\title{
Upper Extremity Prosthetic Technology and Care
}

\author{
Ryan V. Blanck
}

Published online: 15 October 2014

(C) Springer Science + Business Media New York 2014

\begin{abstract}
Significant advances in upper extremity prosthetic care and technology over the last decade have provided clinicians and their patients increased options for treatment and the potential for greater overall functionality. There has been unprecedented focus to the care, research, and technology of the upper extremity amputee in a relative short period of time. Service members injured in OIF/ OEF receiving care for complicated upper extremity amputations have intensified the efforts to improve socket design, interfaces, suspension, and myoelectric function with multiple degrees of freedom as surgical advancement that optimize the intuitive function of the higher level upper extremity amputee. Academic and Government based research groups such as RIC under Dr. Todd Kuiken as well as DARPA and others have generated great interest in the area of surgical advancements coupled with advanced technology. These advanced technologies and unique surgical interventions are intended to provide patients with more intuitive function with myoelectric prostheses along with various advancing technologies of elbow, wrist and shoulder systems. Pattern grasp recognition using and array emg systems for more consistent operation of these advanced robotic technologies is one example that was directly generated from these efforts (Kuiken, Targeted muscle reinnervation a neural interface for artificial limbs, 2014; Dhillon, IEEE Trans Neural Syst Rehabil Eng 13:468-72, 2005). The increased availability of terminal devices with increased functional grasp patterns and conforming grips have provided patients options for more natural functionality of the myoelectric prosthesis for
\end{abstract}

R. V. Blanck $(\bowtie)$

LCPO, Hanger Clinic Specialty Center, 3555 Erickson St., Gig

Harbor, WA, USA

e-mail: rblanck@Hanger.com all levels. With all of these advancing technologies the understanding of optimized upper extremity amputee patient care is more important than ever before. Prior to utilization of these advanced technologies each respective clinician involved in the process must have a firm grasp of the protocols for a well-developed upper extremity plan of care for any patient. A well-educated and experienced clinical understanding for this particular patient group is vital to future success of the patient. Appropriate incorporation of optimized prosthetic design, patient education with a comprehensive team approach coupled these advanced technologies can prove challenging and require a well-designed and intentional method of patient care.

Keywords Technology · TMR · Pattern grasp recognition . Transhumeral $\cdot$ Partial hand $\cdot$ Rehabilitation $\cdot$ Silicone

\section{Introduction}

The last 10 years as brought unprecedented focus to the care, research, and technology of the upper extremity amputee. Treatment centers for the service member injured in OIF/OEF receiving care for complicated upper extremity amputations have intensified the efforts to improve socket design interfaces, suspension, myoelectric function with multiple degrees of freedom as surgical advancement that optimize the intuitive function of the higher level upper extremity amputee. The efforts of groups such as RIC under Dr. Todd Kuiken and others have generated great interest in the area of surgical advancements that provide patients with more intuitive function with myoelectric prostheses along with various advancing technologies of elbow, wrist and shoulder systems as well as pattern grasp EMG systems for more consistent operation of these 
advanced robotic technologies have been directly generated from these efforts [1••]. The availability of terminal devices with increased functional grasp patterns and conforming grips have provided patients options for more natural functionality of the myoelectric prosthesis for all levels. With all of these advancing technologies the understanding of optimized upper extremity amputee patient care is more important than ever before. Prior to utilization of these advanced technologies each respective clinician involved in the process must have a diverse grasp of the entire scope of the upper extremity amputee rehabilitative process. When developing an upper extremity plan of care for any patient a well-educated and experienced clinical understanding for this particular patient group is vital to future success of the patient.

\section{Precursors to Implementation of Prosthetic Technologies}

The Vast majority upper extremity amputations are the result of trauma and the associated complications. While some patients require amputation from various forms of cancerous conditions the most common cause for patients are those who sustain these injuries while working in industrial environments, boating accidents, motorcycle, and vehicle accidents, while operating farming equipment or combat or military training related injuries. All of these various mechanisms can prove to be a challenging rehabilitative process for the patient and their family. Functional outcomes with prosthetic use can vary greatly and are dependent on many key influencing factors. Operative care, rehabilitative care, occupational therapy, and prosthetic care and design all are the variables that are part of long term functional ability of an upper extremity amputee and utilization of a prosthesis. When burns are the amputation cause, the difficulty of the rehabilitation significantly increases. Often the burn related amputation will still maintain the presence of tissue and bone characteristics that complicate the fitting of a prosthetic device. These characteristics often include grafted skin, creative muscle flaps to attained surface coverage, skin adhesions, and continuous tissue breakdown [2•]. Because of the difficulty of these cases, this specific population may experience the most measurable benefit from a multi-disciplinary approach to the upper and lower extremity amputee rehabilitation system. Severe thermal and electrical burns can result in amputations with similar characteristics even though the mechanism of injury is different. Electrical versus thermal related burn injuries may present with several key differences. Electrical burns as result of highvoltage contact are reported to be associated with longer hospital stays and more complex surgical procedures [2•].
When these electrical injuries have been treated with an early fasciotomy, there is an increased incidence of developing deep venous thrombosis and requiring an amputation. There is also an increased prevalence of deep tissue destruction, nerve damage, severe ischemia, and delayed hemorrhage that may compromise future fittings, particularly in the upper extremity [2•].

If a myoelectric system is desired, nerve compromise can result in not only strength and range of motion issues but may also present with reduced efficiency of the EMG signals needed for myoelectric control systems. There also may be possible cognitive issues related to entry or exit of the high voltage electrical injury. Many of the cases have bilateral upper extremity involvement due to the natural tendency for dual hand grasping of live electrical wires for individuals working around high voltage. There is an increased potential for lower extremity damage associated with entry and exit areas of high voltage electrocution injuries. One retrospective study showed that in flame, contact, or fluid burns free flap coverage was performed as a secondary procedure and thus had a higher success rate suggesting that timing of the procedure was correlated to the successful outcome. In terms of the decision to amputate, it is shown that early identification of non-salvageable limbs can decrease infections and improve the mortality rate.

From my personal experiences with this particular patient population as well as upper extremity amputee care as a whole I believe the most successful rehabilitation outcome will progress when the team has a collective understanding of upper extremity surgical procedures, rehabilitation, and prosthetic prescription, technology and training. Team members should have the ability to draw from past experiences as well as a realization that each and every case may require new or varying prosthetic design adaptations that are unique to the patient needs. Communication from each team member will play a crucial role in the progression of the patient's overall functional abilities

In addition, traumatic and or burn related amputations are often combined with other associated injuries that contribute to the complicated poly-trauma nature of the care. In the last decade a significant number of upper extremity amputations secondary to OEF/OEF based combat and training injuries have required rehabilitation within the Department of Defense and all eventually need care and services to be coordinated for them by the VA system or private sector. Access to this care as an amputee with compromised sometimes complex upper extremity amputations may not always prove to be simple to locate. Even today, some areas of the country may be challenging to locate access to appropriate prosthetic care as well as associated ongoing or necessary rehabilitation services and 
availability of current technologies. Under these circumstances, the patient should be educated to be a voice and knowledgeable about needs related to their care and be educated on socket design, interfaces, componentry, and associated technology. Having this knowledge will help them to successfully identify and engage with their future care providers.

\section{Focusing on Maximizing Strength and ROM}

Range of motion limitations often occur as the surface and underlying soft tissues are adherent and have lost mobility and elasticity. This is especially true the presence of skin grafts. ROM deficits will be particularly limiting in the upper they are short of functional range [2•]. Some limitations can be adequately addressed with appropriate choice of terminal device, wrist system, as well as elbow and shoulder joints when applicable. Technology is advancing in this area and there are improved options that are already commercially available to aid in this issue as well as systems that are close to commercial availability that provide various forms of myolectric wrist flexion and extension, internal and external elbow rotation and motorized glenohumeral function for the shoulder level amputee. The issue of limited ROM is more compounded with bilateral involvement of the upper extremity. Maximizing ROM by way of therapies as well as strengthening programs for the elbow and shoulder are fundamental aspects of rehabilitation that have sometimes the greatest long term impact on patients successful utilization of their prosthetic systems. There is many times significant attention to maximizing ROM of the lower extremity amputee but maximizing ROM and upper extremity strength of the upper extremity amputee is equally important.

\section{The Concept of Limb Desensitization}

An area of rehabilitation that can occasionally be missed or not addressed enough is early focus by team and patient on the limb desensitization process. Many patients develop an early tendency toward avoiding contact or even viewing their healing limb soon after their amputation. With this tendency there may be increased possibility for hypersensitivity and prove challenging to find comfort with interfacing with the initial prosthetic system. Volume control and appropriate well fit compression systems can aid in the desensitization process as well as help the patient to interact with their new amputation early and directly. The independent use of shrinkers and liners can prove to be a great initial exposure into the patient's prosthetic use that may just lie ahead for them.
Basic limb ROM and general contact, and manipulation can and should be started very early in the post-operative stage [3•]. Since this early limb desensitization process can be uncomfortable, the discomfort can be eased by performing limb manipulation over and through the compression garment or liner. This also prevents interference with the various wound modalities such as medications and dressings that are being implemented on the skin. At times, custom-designed silicon interfacing has been required even at the pre-prosthetic early stage of treatment due to the shape, ROM, and state of skin integrity of the residual limb. The custom interfaces can be molded, fabricated, and fit on site so as to expedite the process and maintain the treatment progression for the patient.

\section{Patient Education and Future Goals}

Even though it may be very early in the healing phase of rehabilitation, many patients express their desire for the most advanced upper extremity prosthetic system possible. It is important to help them understand that the initial prosthesis may have advanced technology or be a more simple design, and often is some combination of both. Explanation of the reasoning behind the component choices will require that the patient trust the prosthetist and the treating team. Developing an early rapport with the patient and family will give them the confidence that the treatment team can be trusted to keep the patient's best interest at the center of the prescription development for the first limb. The prosthetic options seem overwhelming and many times difficult to fully grasp. The input to the patient can come by way of searches on the web, other well-meaning patients, and publications. It is very important the treating prosthetic team provides appropriate information and be a true support and educational resource and clarity of these options for the patient and family.

\section{Goals for Medical Justification with Prosthetic Care and Associated Technologies}

The focus of advancing prosthetic technology and associated research to provide future benefit to the patient can prove challenging in regards to the medical justification process. Various advancements have occurred even within the last five years that still are not yet considered standards of care though research has already show benefit to the patient. In addition to the normal pre-prosthetic goals and education, the preparation for medical justification can begin as early as the day of injury. Education on medical justification includes all treatment providers involved with care of the patient. Experiences and findings of each team 
member can provide valuable information when formulating a thorough and appropriate medical justification. This is true not only for the initial prosthesis but those that will follow soon after. Many insurance carriers require that a typical upper extremity amputee first utilize standard conventional systems prior to a myoelectric or hybrid system. The main reason for this are related to cost as well as the understanding that in the past many upper extremity amputees put aside the use of their first prosthesis. Certain cases may require more advanced upper extremity myoelectric technology earlier than some patients insurance carriers are accustomed to working with. The more clinical information coupled with published evidence that can be provided to the insurance carrier, the more likely the recommended and prescribed prosthesis will be covered. The patient's potential use of the device in early phases of rehabilitation as well as community reinntegration, selfcare and even returning to an appropriate work environment are all aspects that should be detailed when developing an appropriate medical justification for any device or technology whether simple or advanced.

\section{Upper Extremity Prosthetic Design Considerations}

Considerations with regard to socket design and fabrication as well as the type of prosthetic control system, suspension, wrist, and terminal devices are paramount when addressing ROM limitations and maximizing optimal function that will facilitates patient independence with the prosthesis. In the author's experience, the design of the upper extremity prosthesis must allow for self-care such as toileting, eating, driving, and using a cell phone. If the prosthesis does not assist in the independence of these activities, the patient will seek to find ways to accomplish those tasks without a prosthesis, even as a bilaterally involved upper extremity amputee.

Although independence without a prosthesis is helpful, if a caregiver is needed only because of the inadequacy of the prosthetic device, this issue should be readdressed to optimize fit and function and education. This independence begins with the ability to donn and doff the limb. Problem solving the donning and doffing methods may come from discussions and trial and error between the patient, prosthetist, and occupational therapist.

Limitations of range of motion also influence the decision process over the type of prostheses best suited for the patient. Conventional or hybrid cable operated prosthesis requires significant range of motion at the shoulder and glenohumeral joint for both the affected and contralateral side. Although it is common for conventional or body-powered prostheses to be prescribed first due to reduce costs, the amputation that involves burned and grafted skin or grafted skin covering the proximal intact joints may be most successfully fitted as a myoelectric system.

In many cases, the control system harnessing of a bodypowered limb can be abrasive and very uncomfortable as well as limit the available ROM that is already compromised from issues such as heterotrophic ossification and skin adhesions. Although very costly to begin with, a full myoelectric system can provide a patient greater ROM of the intact limb as well as more efficient range of motion of a myoelectric elbow or wrist. In time, the patient's ROM may increase and the skin may improve in integrity enough to allow operation of a conventional or body-powered prosthesis without difficulty.

As the initial prescription is developed, the cognitive load associated with complicated control systems must be considered. Each aspect of the prosthesis can subject the patient to an increased level of thought required to properly use and operate the device as designed. A balance must be sought that allows the patient experience optimal functionality and an overall sense of success. If the cognitive load is too great, the likelihood of potential of functional failure may increase. One example of reducing the cognitive load would be to program a myoelectric prosthesis or even a TMR system to have standard hand open and close signals but initially keep the wrist unit powered off until the patient masters isolated hand function. Once this is successfully demonstrated, the wrist unit can be powered on and programmed to allow the patient a stepwise progression in the rehabilitation process. Setting reasonable goals with incorporation of activities and prosthetic technology allows the patient to have small victories and accomplishments during the rehabilitation process that keeps them engaged and encouraged by their positive progression in function.

As the first prosthesis is designed, all involved parties need to understand that it is basically a starting point to build from for the future. This process may prove the initial test system to also be exactly what is best for the patient in the long term. Some of the early discussion points can be as simple as the type and location of the power switch, socket alignment angles, terminal devices choices, programming and mode of operation, or even entire the socket design. The test system is usually fabricated with temporary heat moldable plastics to allow for adjustment of volumes changes, pressure distribution modifications, limb length, and functional socket angles. The arm is designed to be used in therapy and outside the facility in real-world situations. I find that if at all possible a take home test system can be very beneficial prior to finalizing and going on to s definitive design. 


\section{Decisions on Level of Amputation}

The process for determination of amputation level can be quite different from one center to another. There are various influences on this decision which include and the training of the surgeons, prosthetists, physical medicine physician, and therapist will play a role in this process. The choices of technology can also now play a role in this decision process. The requirement of length for electronic wrist rotation or advanced or even emerging systems such as the DEKA arm, Michelangelo hand systems or partial hand systems such as the prodigit or Vincent hand systems have already been part of discussions in certain treatment centers when determining what surgical options might be most prudent for optimizing function in the future. These examples of technology influencing surgical and rehabilitation treatments are already occurring now but with continued advancement in technologies these types of conversations will continue to grow as these various systems continue to develop and are validated with treatment and research. A more simple example of surgical choice to maximize potentials may be in the case of donning a wrist disarticulation prosthesis over a transradial system, being more efficient and easier for a bilateral, particularly when the other side is transradial or higher. Another example may be a shoulder disarticulation being preferred over a short transhumeral if the patient has significant glenohumeral ROM restrictions that are anticipated to limit their ability to utilize prosthesis. A more proximal amputation may also be considered if EMG signals are unobtainable at a lower level. The emerging availability of compound electronic wrist systems humeral rotators and electronically controlled shoulder systems are a few examples of technologies that will certainly play a part in surgical level decisions in the future.

\section{Socket and Interface Design}

The socket system is the fundamental base for success for control of the entire prosthesis. As previously mentioned, varying challenges or anatomy and compromised surface tissues following upper extremity amputation must somehow now interface with various prosthetic materials and minimize various potentials of shear, slip, torque, pressure, and distraction. The advances of technology don't necessarily mean lighter weight but actually the opposite. The increased degrees of freedom, power, and speed of myoelectric systems come with increased weight that must be controlled and addressed with sometimes very unique and supportive and even dynamic socket designs. The most common choices of interface options include prefabricated roll-on liners, custom-designed roll-on liners, direct socket

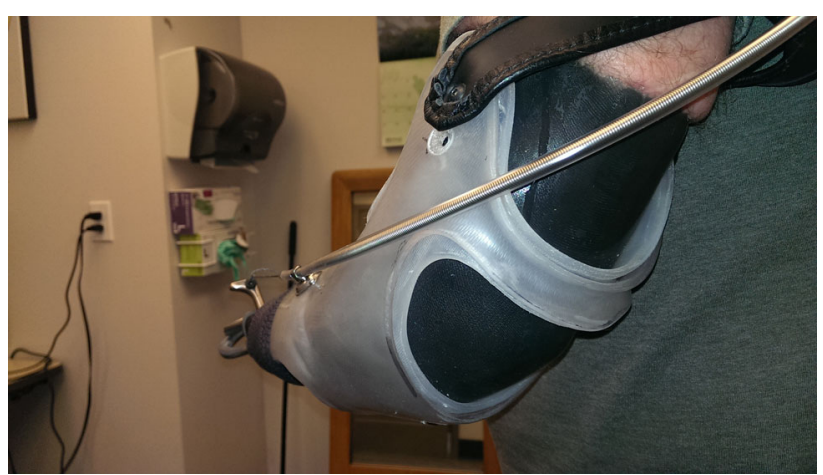

Fig. 1 Silicone inner socket interface

interfacing, or even custom static inner silicon liners. Each of these have the potential to work, but the decision once again is made on an individual case basis being influenced by past experience of the prosthetist and other members of the team. As an example, the prefabricated liners range in elasticity, co-efficient of friction, and material, all of which must be matched with the patient limb characteristics.

Suspension of the prosthesis can come in a single form or multiple forms working together. In general, the prosthesis should be suspended using as much skin surface area as possible. Focalized suspension, as in the case with pin locking and sealing ring liners, may be prone to exhibit increased levels of skin friction over tenuous or fragile skin due to the distally located suspension mechanisms of these systems (Fig. 1). Full contact suction utilizing a static custom silicone liner can be done at the partial hand, wrist disarticulation, trans-radial, elbow disarticulation, and transhumeral levels. This provides suction suspension over the length of the limb yet protects the skin with the pliable silicone.

In the author's experience, this custom molded liner is fabricated using low durometer silicone that can also incorporate the electrodes if a myoelectric system is desired. The custom static silicon inner liner is fabricated using materials that most closely mimic the elasticity and integrity of normal skin and tissue. This material works very well in cases of delayed healing as well as tenuous tissue with or without the use of light friction reducing dressings on the limb. It can also accommodate moderate levels of limb volume fluctuation as well as easing the difficulty of donning the prosthesis over irregular shapes and compromised or even open skin. The socket system is donned using readily available lubricants. This custom interface can even be used as a shrinker prior to full fabrication of the socket (Fig. 2).

Once the appropriate inner socket interface is provided, the outer structural shell and socket is begun. The outer socket will provide the structural integrity necessary to properly transmit forces from the limb to the prosthesis and provide an attachment basis for the other prosthetic 


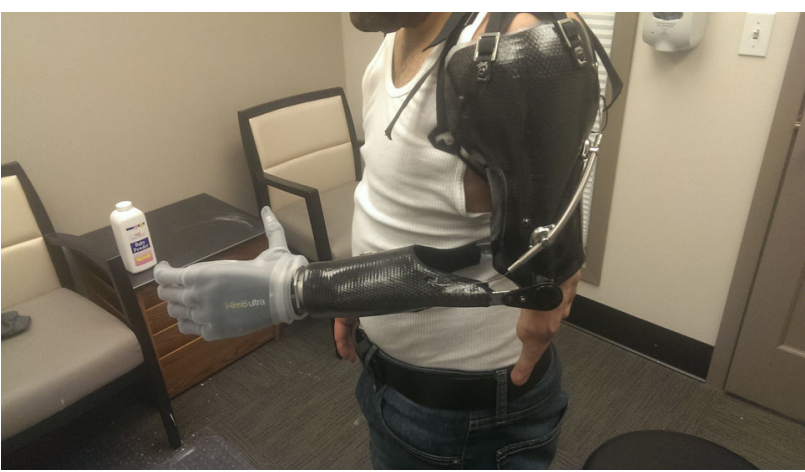

Fig. 2 Congenital above elbow hybrid system with conforming grasp hand

components. Rotational control of the prosthesis is a significant problem that can be addressed in two ways. The use of significantly higher proximal trimlines may be necessary to add control of the prosthesis on the more proximal amputations. Rotational control must also be addressed within the body of the socket by careful design of pressures that will compress and displace the soft tissue in relationship to the bony anatomy. Most of the socket stability should come from the design of the contours that pressure the tolerant areas of the limb then secondarily by way of suspension sleeves and harnessing designs. These load bearing regions must be very well thought out. The obvious unloading of bony anatomy is required but with burned and grafted tissue, high focal pressures may result in undesired skin breakdown. Often there is little underlying subcutaneous tissue for protection of underling muscle and tendons. If myoelectric control is desired, design consideration needs to be given to prevent excess pressure that can degrade the thin skin and develop into a skin ulcer at the myosite. The socket design in the case of targeted muscle reinnervation (TMR) with up to 6 standard electrodes and now the use of pattern grasp technology with an array of electrodes to maximize intuitive prosthetic function both are examples of systems that require maximally stable socket designs. These system do not tolerate shifts in socket positions or any form of translation [3•] (Fig. 3). The socket design, interface, and overall suspension is paramount in successfully function for amputees with TMR procedures as well as standard use of EMG signals. The development use of implantable electrodes is directly related to the challenges of socket design and optimized function of EMG signals within a socket and limb interface.

\section{Elbow Options}

With amputations at or above the elbow, there are several variations in prosthetic elbow choices. The body-powered

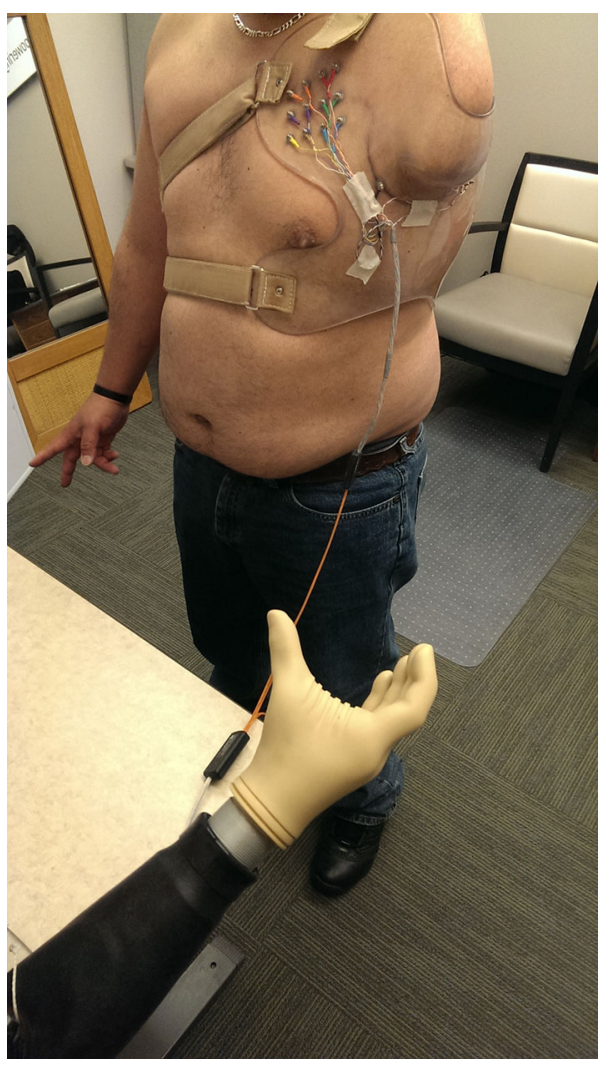

Fig. 3 Coapt pattern grasp TMR system

elbow systems require a significant amount of scapular and glenohumeral ROM for full utilization. Various combinations of shoulder motion are captured by the harness system to produce combinations of elbow flexion, terminal device operation, and positional elbow locking. A fully body-powered system requires the greatest shoulder range for operation of both the mechanical elbow and terminal device operation. While a mechanical elbow may require the largest degree of strength and range, it is also places the least cognitive load on the patient. This body-powered elbow is the most commonly prescribed prosthetic elbow for a new prosthetic user. This could be someone with a recent amputation or one who has not previously chosen to use a prosthesis. It is a common approach to prescribe a conventional body-powered above elbow prosthesis to begin the rehabilitation process and establish a prosthetic use pattern.

Once the patient demonstrates successful use of this simple prosthetic design, they may be considered for a hybrid or fully electronic prosthetic system. This approach is reasonable as long as a body-powered system is still the right choice. If there are other limitations to the use of harnessing and body power, use of a conventional prosthesis will likely not be successful and the patient could reject any use of a prosthetic device. 


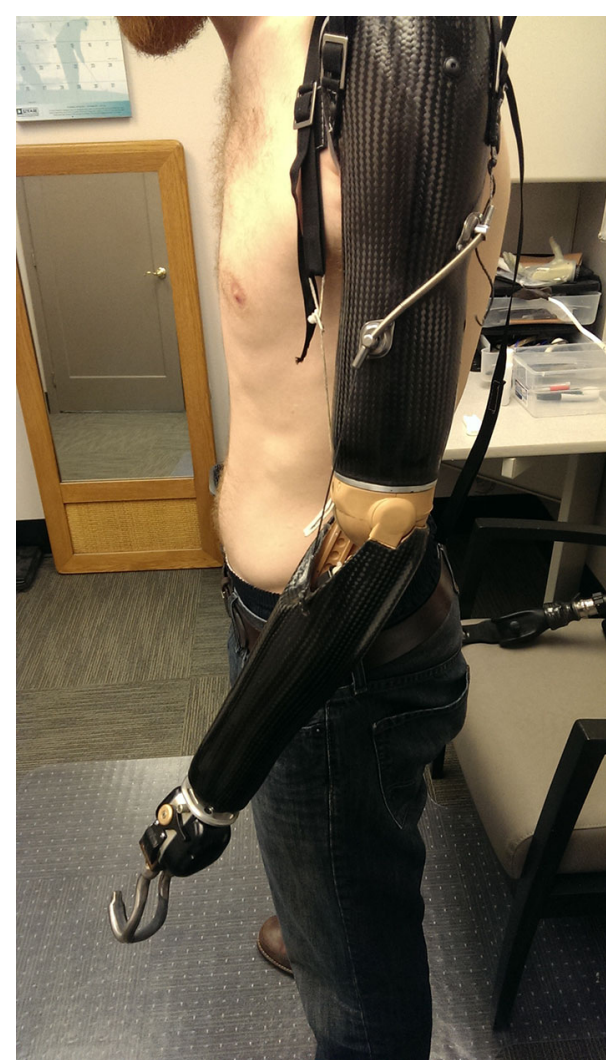

Fig. 4 Transhumeral hybrid system with electronic terminal device (ETD)

When there are physical limitations for implementation of body power, a hybrid design should be considered. A hybrid system most commonly includes a mechanical elbow with a myoelectric hand and wrist rotator. The hybrid prosthetic system uses a traditional harness for body-powered activation of elbow movement in combination an electronically operated wrist and terminal device function (Fig. 4). The terminal device and wrist can be EMG or mechanical switch controlled. The compromise of the introduction of electronics include an increased level of cognitive load and associated training, moderate increase in weight associated with the electronic wrist, terminal device and battery, and significant cost increase.

If appropriate, a fully electronic system can be provided that includes electronic function of all aspects of the prosthesis including the elbow, wrist, and hand. It may operate with use of 1-2 electrodes as well as other electronically activated pull switches, forces sensors, or cable tension activated transducers. The fully electronic above elbow prosthesis requires the least amount of harness activated function and shoulder range of motion for activation of the desired function. This system will be the heaviest and most costly. In the burn amputation population, the traditional mindset of demonstrating mastery of a body-powered prosthesis prior to considering electronics may not actually be in the best interest of the patient for several reasons [2•]. The sheer forces across the burned skin and grafted tissue caused by body-powered harness systems are rarely tolerated within the first 3-6 months of prosthetic use. Many of these patients do not have adequate range of motion or ability to generate joint power to operate a conventional or hybrid elbow system. In the absence of joint range and strength, they may still be able to operate a fully electronic elbow system with EMG activation or another form of electronic operation of the elbow, wrist, and hand.

If this system is chosen, careful attention must be paid to appropriate socket design, skin interface, and the secondary suspension harness that will enable the patient to tolerate use of a complicated, heavy limb. Many of these elbow systems allow for gradual progression of function in the early phases of rehabilitation and training by incorporating wireless programming options through an on-board microprocessor. This gradual progression of the complexity of the system allows for reduced cognitive load in early training and advancement of the elbow function when appropriate.

One additional challenge to be aware of is the effect of the elbow choice on limb length and matching anatomical elbow centers. The elbow disarticulation amputation has limited elbow options. Although it can be a very functional level, it will require the use of mechanical outside locking hinges to keep bilateral elbow centers equal and maintain some symmetry in appearance to the sound side. Outside locking hinges can either be used in a conventional bodypowered prosthesis or even the hybrid version depending on the patient's needs. Although generally not cosmetically acceptable, a fully electronic above elbow system may be warranted to allow for optimized levels of function and independence due to limited ROM, levels of skin integrity. Occasionally, if extended wheelchair use is necessary due to lower extremity complications, a powered system may be necessary overcome the limitations of using body power while seated.

\section{Wrist and Hand Options}

In this patient population, normal joint range of motion is rarely demonstrated. It is also very common to encounter bilateral upper extremity involvement where the sound contralateral side has amputation or is intact but has compromised strength and range of the hand, wrist, elbow, or shoulder. Given this scenario, the choice of terminal device and wrist unit are even more important.

A primary goal with any prosthesis, whether body powered or myoelectric, is to enable the patient to reach midline for eating and toileting purposes. However, this basic fundamental principle is achieved does not really 


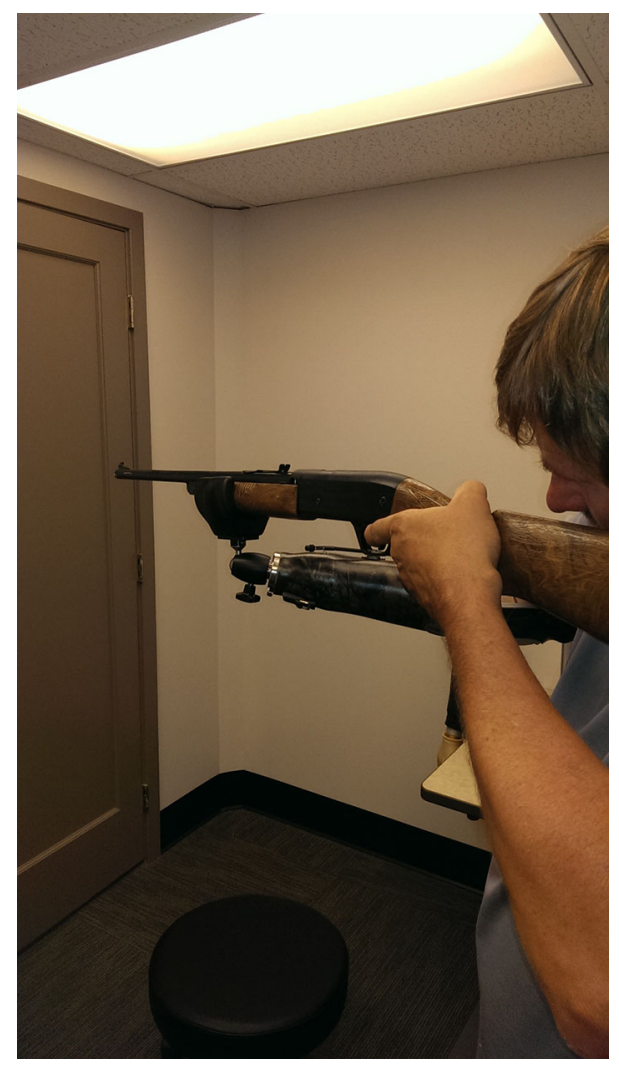

Fig. 5 Lamprey recreational adapter

matter, but there are plenty of options available to the well informed treatment team. This is not to minimize the importance of other tasks that facilitate independence, but these are milestones of the early rehabilitation goals.

Terminal devices will be manually or electrically controlled. The goal is to select a mechanical or myoelectric terminal device and wrist combination that allows for the greatest function to facilitate activities of daily living. There have been minimal advances in technology for the mechanically operated terminal device and wrist but they remain a standard of care due to affordability and durability. The standard conventional options include the traditional opening and closing devices that are hand or hook based and offer various locking mechanism for a sure grip on an item. There are also wrist flexion systems that are a separate component or may be integrated into the terminal device. A few cable operated mechanical wrist rotation systems are produced, but their use and function is limited.

If a myoelectric device is desired, one must overcome the challenge of gaining access to these advanced systems. They require specialized skill by the provider and will incur significant cost that must be justified to the payer. Although not for everyone, their benefits are numerous. As in many of the elbow and knee systems, the terminal devices have microprocessor control and blue tooth programmability. The

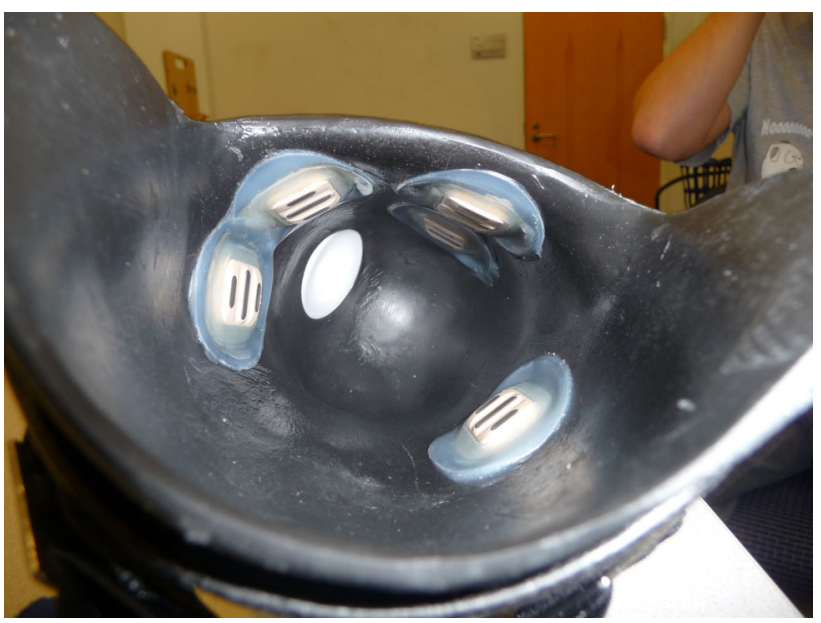

Fig. 6 TMR transhumeral inner socket electrodes

prosthesis and associated terminal device can be configured to best meet the needs of the patient with real time adjustments of EMG gain and thresholds, speed, grip force, and control patterns. One of the most significant advances is the option to have multiple gasp pattern choices on a single myoelectric hand system (Fig. 5). This has allowed the patient to expand the grip options available to them with a simple control sequence that can be performed in less than a second. Some of these additional patterns can include pinch grasp, three jaw chuck, lateral pinch, and power grip. In addition to these current advances in technology, various forms of electronic wrist flexion and extension as well as ulnar and radial deviation will soon be available [4•].

With advances in the electronic hardware for the upper extremity, the methods of purposeful control become more complicated, The is a significant focus on research related to developing proprioceptive feedback, and neural interface by way of surgical modification of nerves such as TMR, emg implants, cortical implants, and pattern grasp recognition [2•, 4•] (Fig. 6).

Even with all the current advanced options there is not one perfect terminal device or control strategy that will facilitate every activity one desires. When feasible, it is best to allow the patient to trial multiple terminal devices outside of the clinical setting. This will help them develop an understanding of the capability of each and how a particular device may be best suited to their needs.

As is continually experienced with lower extremity prosthetic advances, access to this technology must be supported by evidence of the outcome benefits or request for insurance coverage will usually be denied. Amputees desire to function beyond even the normal activities of daily living. It can also be important to discuss what additional activities they may choose to be involved with that traditional terminal devices may not accommodate (Fig. 7). The options to 


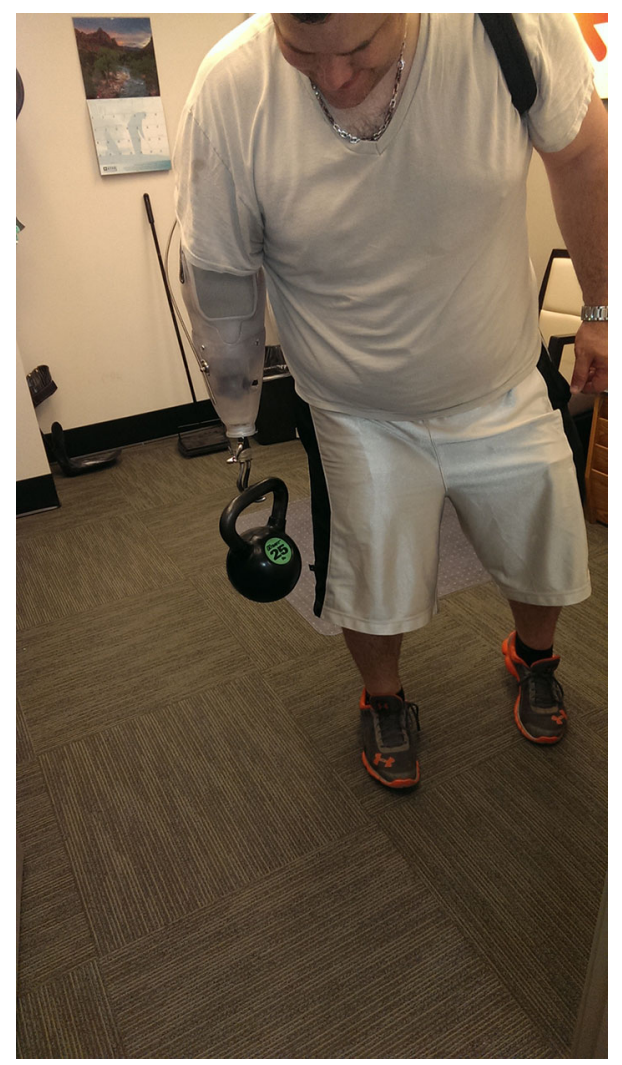

Fig. 7 Suspension test

patient for adapters and additional mechanisms for recreational or activity specific focuses have widely expanded over the last 10 years. Medical justification for these systems can be challenging but successful when the specific need and benefit is well documented.

\section{Follow up and Long Term Care}

When the patient is nearing completion of their formal rehabilitation, they will need to be educated on the importance of self-advocacy. If they have been treated at an advanced medical care center, they most likely had the clinical services of a highly skilled rehabilitation team who are very familiar with the upper extremity rehabilitation process. Once they have completed therapy and been discharged from care, the need for a skilled prosthetist remains. The prosthetic socket will need to be replaced as the limb undergoes expected changes, and the powered components will need service and adjustment. If they have been provided and trained with a complex powered system, there may be some difficulty in having service and maintenance performed in their local community. It is reasonable to consider the provision of basic back-up prosthesis to be used in the likely situation where the complex powered limb is in need of service as well as providing simple customized assistive devices for optimal use of daily activities.

\section{Conclusion}

The fundamentals of care are still the most important aspects of provision of care for the upper extremity amputee. Technology is advancing and will continue to do so in the years to come. This is an exciting time for this field of care as well as the patients it serves but emphasis on the basics of postoperative care, education, socket design, and interface and overall support may still be more important than the most advanced hand or elbow technologies. The team treating the upper extremity amputation patient needs to continue sharing the advances that are being made within each of discipline supporting the process. Developing relationships amongst each respective care giver and the center of the focus in the patient the technologies that are implemented will prove much more successful as well as useful to the patient. Practicing with this in mind, it will always be in the patient's best interest to have a working knowledge of each of the disciplines involved. The future for upper extremity care and technologies looks very promising, but the utilization for these systems is not the same for every patient. The clinicians involved in this process need to be willing to provide a patient with technology and training in an organized and structured way while maintaining flexibility when challenges arise.

\section{Compliance with Ethics Guidelines}

Conflict of Interest R. V. Blanck declares no conflicts of interest.

Human and Animal Rights and Informed Consent This article does not contain any studies with human or animal subjects performed by any of the authors.

\section{References}

Papers of particular interest, published recently, have been highlighted as:

- Of importance

•- Of major importance

1. $\bullet$ Kuiken T, Schulz Feauser A, Barlow A, Targeted muscle reinnervation a neural interface for artificial limbs, vol 1. Boca Raton: CRC Press; 2014. p 4-6. This is a reference detailing the new technologies now available for all levels and segments for the upper extremity amputee that has been potentially enhanced by TMR procedures.

2. - Ward RS, Hayes-Lundy C, Schnebly WA, Saffle JR. Prosthetic use in patients with burns and associated limb amputations. J Burn Care Rehabil. 1990;11(4):361-4. This is a reference that details 
optimization of prosthetic use within the burn and limb amputations.

3. - Schneider JF, Holavanahalli R, Helm P, Goldstein R, Kowalski $\mathrm{K}$. Contractures in burn injury: defining the problem. J Burn Care Res. 2006;27(4):508-14. This is a reference to the importance of socket interface, design and suspension in relationship to the complex treatment of burned and grafted tissue.
4. - Dhillon GS, Horch KW. Direct neural sensory feedback and control of a prosthetic arm. IEEE Trans Neural Syst Rehabil Eng. 2005;13(4):468-72. This is a reference detailing the advanced surgical procedures and enhanced function of the upper extremity prosthesis for patients with high level upper extremity amputations. 